\title{
High basal level of autophagy in high-altitude residents attenuates myocardial ischemia-reperfusion injury
}

\author{
Yijie Hu, MD, Qi Sun, MD, Zhiping Li, MD, Jianming Chen, MD, Cheng Shen, MD, Yi Song, MM, and \\ Qianjin Zhong, MD
}

\begin{abstract}
Objective: Hypoxia can induce autophagy, which plays an important role in cardioprotection. The present study tested the hypothesis that patients with congenital heart disease living at a high altitude could resist ischemiareperfusion injury better than those at a low altitude, through elevated basal autophagy by chronic hypoxia.
\end{abstract}

\begin{abstract}
Methods: Twelve Tibetan patients residing at a high altitude of $>3000 \mathrm{~m}$ and 12 Han patients residing at a low altitude of $<500 \mathrm{~m}$ with simple atrial or ventricular septal defects were prospectively recruited. All patients underwent cardiopulmonary bypass, maintaining a flow rate of approximately 2.4 to $2.8 \mathrm{~L} / \mathrm{min} / \mathrm{m}^{2}$ and mean arterial pressure of $\geq 40$ to $60 \mathrm{~mm} \mathrm{Hg}$. Myocardial ischemia-reperfusion injury between the 2 groups was compared using cardiac troponin I, brain natriuretic peptide, hematoxylin eosin staining, and the terminal deoxynucleotidyl transferase dUTP nick end labeling test. Autophagy-related proteins microtubule-associated protein 1 light chain 3 II (LC3II), Beclin1, and lysosomal-associated membrane protein 2 (LAMP2) and their upstream protein BCL2/adenovirus E1B 19-kDa protein-interacting protein 3 (Bnip3) were evaluated with Western blotting.

Results: The maximal cardiac troponin I concentration and increasing $\mathrm{x}$-fold of brain natriuretic peptide in the high-altitude group were obviously lower than those in the low-altitude group $(3.10 \pm 0.77 \mathrm{vs} 7.10 \pm 2.28 \mathrm{ng} / \mathrm{mL}$ and $2.51 \pm 0.94$ vs $14.66 \pm 6.83$, respectively). The preoperative and postoperative levels of LC3II, LAMP2, and upstream Bnip3 in the high-altitude group were obviously greater. No difference was found in the Beclin1 level between the 2 groups at baseline or ischemia-reperfusion.

Conclusions: Patients living at a high altitude with congenital heart disease resisted ischemia-reperfusion injury during cardiac surgery better than those at a low altitude, possibly through elevated basal autophagy induced by chronic hypoxia. (J Thorac Cardiovasc Surg 2014;148:1674-80)
\end{abstract}

It has been previously reported that the prevalence of congenital heart disease (CHD) in residents living at a high altitude is about $1.37 \%, 2$ to 3 times that of those living at a low altitude. ${ }^{1}$ According to the Chinese census in 2005 (China's Fifth National Census), the total Tibetan ethnic population was 5,416,021; most of whom live at altitudes $>3000 \mathrm{~m}$. Hence, many Tibetans will require cardiac intervention. With the improvement in healthcare and transportation, many Tibetans with CHD in the Qinghai-Tibet Plateaus will have the opportunity to undergo surgery at the heart center in the lowlands. However, only limited data are available on myocardial

From the Department of Cardiovascular Surgery, Institute of Surgery Research, Daping Hospital, Third Military Medical University, Chongqing, China.

The present study was supported by the National Natural Science Foundation of China (grant 81200182)

Disclosures: Authors have nothing to disclose with regard to commercial support.

Y.H. and Q.S. contributed equally to this work.

Received for publication Jan 23, 2014; revisions received March 19, 2014; accepted for publication March 21, 2014; available ahead of print April 19, 2014.

Address for reprints: Qianjin Zhong, MD, Department of Cardiovascular Surgery, Institute of Surgery Research, Daping Hospital, Third Military Medical University, No. 10 Changjiang Branch Rd, Yuzhong District, Chongqing 400042, China (E-mail: zhongqianjin@qq.com).

0022-5223/\$36.00

Copyright (c) 2014 by The American Association for Thoracic Surgery

http://dx.doi.org/10.1016/j.jtcvs.2014.03.038 ischemia-reperfusion injury during cardiac surgery in high-altitude residents.

The average arterial partial oxygen pressure of habitants in the Qinghai-Tibet Plateaus has been about $57 \mathrm{~mm} \mathrm{Hg} .{ }^{2,3}$ It is well established that chronic hypoxia improved Bcl-2/adenovirus E1B $19-\mathrm{kDa}$ interacting protein 3 (Bnip3) expression, which could increase the autophagy level through inhibition of the mammalian target of rapamycin (mTOR). ${ }^{4}$ Autophagy is an intracellular bulk degradation process, in which cytosolic, long-lived proteins and organelles are degraded and recycled. Autophagy occurs at basal levels but can be further induced by stress, such as nutrient depletion and hypoxia. ${ }^{5}$ It confers resistance against hypoxia, which could make the native population of the Qinghai-Tibet Plateaus to acclimatize to the high altitude and tolerate hypoxia well.

Evidence from in vitro and in vivo animal studies has suggested that autophagy can play a significant role in myocardial ischemia-reperfusion injury. ${ }^{6-10}$ In particular, basal levels of autophagy are important for maintaining cellular homeostasis and protecting cells against damaged or dysfunctional organelles. Enhancing autophagy can protect against ischemia-reperfusion injury in cardiac myocytes. ${ }^{11}$ However, the availability of clinical data is limited. 


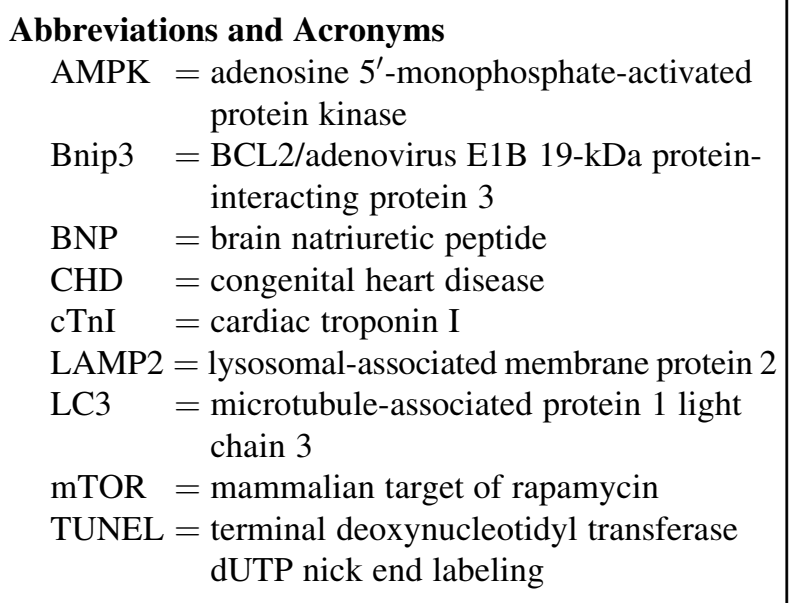

Therefore, we hypothesized that high-altitude patients with CHD could resist subsequent surgical ischemiareperfusion injury during cardiac surgery better than those living at a low altitude by the elevated basal autophagy induced by chronic hypoxia. To assess this hypothesis, the incidence of myocardial ischemia-reperfusion injury of Tibetan patients with CHD was compared with that of patients living at a low altitude. We also investigated the initial autophagy status, its response to myocardial ischemia-reperfusion, and the upstreaming of hypoxiarelated Bnip3.

\section{METHODS}

The Clinical Research Ethics Committee of Daping Hospital, Third Military Medical University (Chongqing, China) approved the present clinical study and the use of human tissue. All participants provided written informed consent before study enrollment. To accurately evaluate the effect of basal autophagy on myocardial ischemia-reperfusion injury, the patients who met the following criteria were included: (1) simple atrial or ventricular septal defect, which could be sewn closed directly within a short and similar aortic clamping time; (2) age 6 to 18 years, lessening age's effect on autophagy; and (3) without organ dysfunction or special preoperative medical history.

A total of 12 Tibetan patients who were undergoing elective open heart surgery with cardiopulmonary bypass were prospectively recruited for the high-altitude group (residence altitude, $>3000 \mathrm{~m}$ ). A total of 12 Han patients were matched as a control group, the low-altitude group (residence altitude, $<500 \mathrm{~m}$ ).

All surgeries were performed with the patient under general anesthesia and with cardiopulmonary bypass, maintaining a flow rate of approximately 2.4 to $2.8 \mathrm{~L} / \mathrm{min} / \mathrm{m}^{2}$ and a mean arterial pressure of $\geq 40$ to $60 \mathrm{~mm} \mathrm{Hg}$ using y a centrifugal pump (MaQuet Rotaflow, Mahwah, $\mathrm{NJ}$ ). del Nido cardioplegia was given as a single $15-\mathrm{mL} / \mathrm{kg}$ dose antegrade. With the cardioplegia passing through a cooling coil in ice, the delivery temperature was $8^{\circ} \mathrm{C}$ to $12^{\circ} \mathrm{C}$. The aortic root pressure was not monitored, but the surgeon monitored aortic root distension closely during delivery to prevent capillary damage from high shear forces with too rapid delivery. Right atrial tissue (about $200 \mathrm{mg}$ ) was harvested before the establishment of cardiopulmonary bypass and 10 minutes after opening the crossclamp. One half of the right atrial tissue was immediately frozen in liquid nitrogen and processed for Western blotting, and one half was fixed in 10\% neutral formalin for immunohistochemistry. Cardiac troponin I (cTnI), a marker of myocardial injury, was tested using the Alere Triage fluorescence immunoassay (Alere, Inc, Waltham, Mass). Brain natriuretic peptide (BNP) was measured using a chemiluminescence immunoassay from a blood sample withdrawn for other routine blood tests. Blood samples were collected for these laboratory tests preoperatively and 6,24 , and 72 hours postoperatively.

\section{Histologic Analysis}

The fixed atrial tissue in $10 \%$ neutral formalin was processed for histologic examination using standard techniques. It was then embedded in paraffin, and $5-\mu \mathrm{m}$ sections were stained with hematoxylin and eosin for histologic evaluation of tissue damage. For a semiquantitative estimation of the tissue damage, the method described by Zingarelli and colleagues $^{12}$ was used. According to this scoring system, the following criteria were used: score 0 , no damage; score 1 (mild), interstitial edema and focal necrosis; score 2 (moderate), diffuse myocardial cell swelling and necrosis; score 3 (severe), necrosis with the presence of contraction bands and neutrophil infiltration; and score 4 (highly severe), widespread necrosis with the presence of contraction bands, leukocyte infiltration, and hemorrhage.

\section{Terminal Deoxynucleotidyl Transferase dUTP Nick End Labeling Assay}

The myocardial tissues embedded in paraffin were sectioned ( $2 \mu \mathrm{m}$ thickness) for the terminal deoxynucleotidyl transferase dUTP nick end labeling (TUNEL) assay with a commercial in situ cell death detection kit (Roche, Mannheim, Germany). According to the manufacturer's protocol, the deoxyribonucleic acid nick ends were visualized with diaminobenzidine. The numbers of $4^{\prime}, 6$-diamidino-2-phenylindole-positive nuclei and TUNEL-positive nuclei were quantified from the average of 3 randomly selected fields/section using Image Pro Plus, version 7.0 (Media Cybernetics, Rockville, Md), performed by a technician who was unaware of the samples' group. The number of TUNEL-positive cells is expressed as a percentage of the total number of cells.

\section{Western Blot Analysis}

Total proteins were isolated from the right atrial tissue samples. The sample was then size fractionated using sodium dodecyl sulfate-polyacrylamide gel electrophoresis and transferred to Immobilon-P membranes (EMD Millipore, Billerica, Mass). The blotted membranes were incubated with antibodies against microtubule-associated protein 1 light chain 3 II (LC3II), lysosomal-associated membrane protein 2 (LAMP2), Beclin1, and Bnip3 (all at a 1:600 dilution; Abcam, Cambridge, UK). After incubation with the appropriate horseradish peroxidase-associated secondary antibodies (Santa Cruz Biotechnology, Santa Cruz, Calif), the signals were visualized using an enhanced chemiluminescence detection system (Amersham Bioscience, Piscataway, NJ). Quantification of the Western blot data was performed by measuring the intensity of the hybridization signals using ImageJ, version 1.42, software (National Institutes of Health, Wayne Rasband, available at: http://rsb.info.nih.gov/ij).

\section{Statistical Analysis}

The data are expressed as the mean \pm the standard error of the mean The difference in the mean between the 2 groups was evaluated using the $t$ test when sample size was appropriate and the population was normally distributed; otherwise, the Mann-Whitney $U$ test was used. Statistical analyses were performed using IBM SPSS Statistics, version 19.0 software (SPSS, Inc, Armonk, NY).

\section{RESULTS}

\section{Perioperative Patient Variables}

No difference was found in age, gender, preoperative hemoglobin, hematocrit, aortic clamping time, mechanical 
TABLE 1. Perioperative variables of patients

\begin{tabular}{lcc}
\hline Perioperative variable & $\begin{array}{c}\text { High-altitude } \\
\text { group }(\mathbf{n}=\mathbf{1 2})\end{array}$ & $\begin{array}{c}\text { Low-altitude } \\
\text { group }(\mathbf{n}=\mathbf{1 2})\end{array}$ \\
\hline Ethnicity & Tibetan & Han \\
Age (y) & $14 \pm 8$ & $9 \pm 2$ \\
Gender & & \\
$\quad$ Male & 5 & 5 \\
$\quad$ Female & 7 & 7 \\
Septal defect & & \\
$\quad$ Atrial & 6 & 6 \\
$\quad$ Ventricular & 6 & 6 \\
Hemoglobin (g/L) & $120.4 \pm 39.2$ & $128.0 \pm 5.0$ \\
Hematocrit $(\%)$ & $35.0 \pm 7.3$ & $34.4 \pm 2.7$ \\
Aortic clamping time (min) & $20.6 \pm 7.8$ & $17.8 \pm 4$ \\
Mechanical ventilation (h) & $6.3 \pm 1.6$ & $5.6 \pm 1.5$ \\
Complications & None & None \\
\hline
\end{tabular}

ventilation time, or the incidence of postoperative complications between the high-altitude and low-altitude groups (Table 1). Because all cases were sewn closed directly using a similar aortic clamping time, few differences in the incidence of myocardial injury was presupposed for the different congenital anomalies (atrial septal defect or ventricular septal defect).

\section{Myocardial Ischemia-Reperfusion Injury}

Molecular marker for myocardial ischemia-reperfusion injury. The preoperative cTnI concentrations in both groups were not more than $0.05 \mathrm{ng} / \mathrm{mL}$. The maximum cTnI concentration was observed 6 hours after surgery, and the cTnI concentration in the high-altitude group was obviously lower than that in the low-altitude group $(3.10 \pm 0.77$ vs $7.10 \pm 2.28 \mathrm{ng} / \mathrm{mL}, P<.05)$. Then, the cTnI concentration decreased gradually, and no obvious difference was observed between low-altitude and highaltitude groups at 24 hours $(0.83 \pm 0.61$ vs $1.39 \pm 1.12$ $\mathrm{ng} / \mathrm{mL}, P>.05)$ or 48 hours $(0.33 \pm 0.16$ vs $0.31 \pm 0.09$ $\mathrm{ng} / \mathrm{mL}, P>.05$; Figure $1, A)$.

The baseline BNP concentration in the high-altitude group was significantly greater than that in the lowaltitude group $(211.20 \pm 24.58$ vs $30.50 \pm 24.91 \mathrm{pg} / \mathrm{mL}$, $P<.01)$. In both groups, the BNP concentration had increased 6 hours after surgery and had reached a maximum at 24 hours after surgery. However, no obvious difference was seen in the maximum BNP concentration between the high-altitude and low-altitude groups $(534.20 \pm 224.61 \mathrm{vs}$ $328.75 \pm 75.98 \mathrm{pg} / \mathrm{mL}, P>.05$; Figure $1, B$ ).

BNP release is a marker of increased myocardial wall tension, which will be elevated in patients with disturbed ventricular function. ${ }^{13,14}$ Considering the upregulation of BNP gene expression by preoperative chronic myocardial hypoxia, ${ }^{14}$ increasing $\mathrm{x}$-folds of BNP [(maximum postoperative $\mathrm{BNP}) /($ preoperative $\mathrm{BNP})$ ] were adopted to evaluate the injury of ventricular function. The maximum
BNP concentration in the high-altitude group was $2.51 \pm 0.94$-fold greater than the baseline level. In contrast, the maximum BNP concentration in the low-altitude group was increased $14.66 \pm 6.83$-fold.

Pathologic characteristics of myocardial ischemiareperfusion injury. No obvious differences were found in the preoperative atrial hematoxylin and eosin-staining between the high- and low-altitude groups. However, more severe myocardial injury was found in the ischemic-reperfused atrial section of the low-altitude group with more contraction bands and greater neutrophil infiltration (Figure 2). The damage score for the low-altitude group was significantly greater than that of for the high-latitude group $(3.5 \pm 0.6$ vs $1.8 \pm 0.8, P<.01)$.

A representative TUNEL-stained preoperative section demonstrated relatively fewer apoptotic cells in the low-altitude group than in the high-altitude group $(0.06 \pm 0.02$ vs $0.24 \pm 0.02, P<.05)$. However, TUNELpositive cells were significantly more increased in the low-altitude group than in the high-altitude group $(0.75 \pm 0.07$ vs $0.41 \pm 0.04, P<.05$; Figure 2$)$.

\section{Myocardial Autophagy Status and Its Upstreaming of Hypoxia-Related Bnip3} Myocardial autophagy status before and after ischemiareperfusion injury. To evaluate the relationship between myocardial ischemia-reperfusion injury and autophagy, the levels of LC3II, Beclin1, and LAMP2, the 3 main autophagic molecular markers, at baseline and the ischemia-reperfusion point were measured.

Intracellular LC3 underwent a conversion from the LC3I to the LC3II isoform, which is specific for autophagosomes. Hence, the production of LC3II has been established as an indicator of autophagy induction. Ischemia-reperfusion injury activated cardiomyocyte autophagy in the high- and low-altitude groups both. However, the preoperative LC3II level in the high-altitude group was obviously greater than that in the low-altitude group. Ischemia-reperfusion provoked a more significant increase in LC3II in the highaltitude group than in the low-altitude group (Figure 3).

The level of Beclin1 was then detected, because Beclin1-induced autophagy has been reported to exacerbate myocardial ischemia-reperfusion injury. In contrast to the obvious findings of Beclin1 elevation in ischemiareperfusion injury, it had decreased obviously for both groups in our study. However, no difference was found in the Beclin1 level between the high- and low-altitude groups at baseline or the ischemia-reperfusion point (Figure 3).

The level of LAMP2, a critical determinant of autophagosome-lysosome fusion, in the high-altitude group was obviously higher than that in the low-altitude group at both baseline and the ischemia-reperfusion point. In accordance with a previous report, it declined obviously in both groups (Figure 3). 

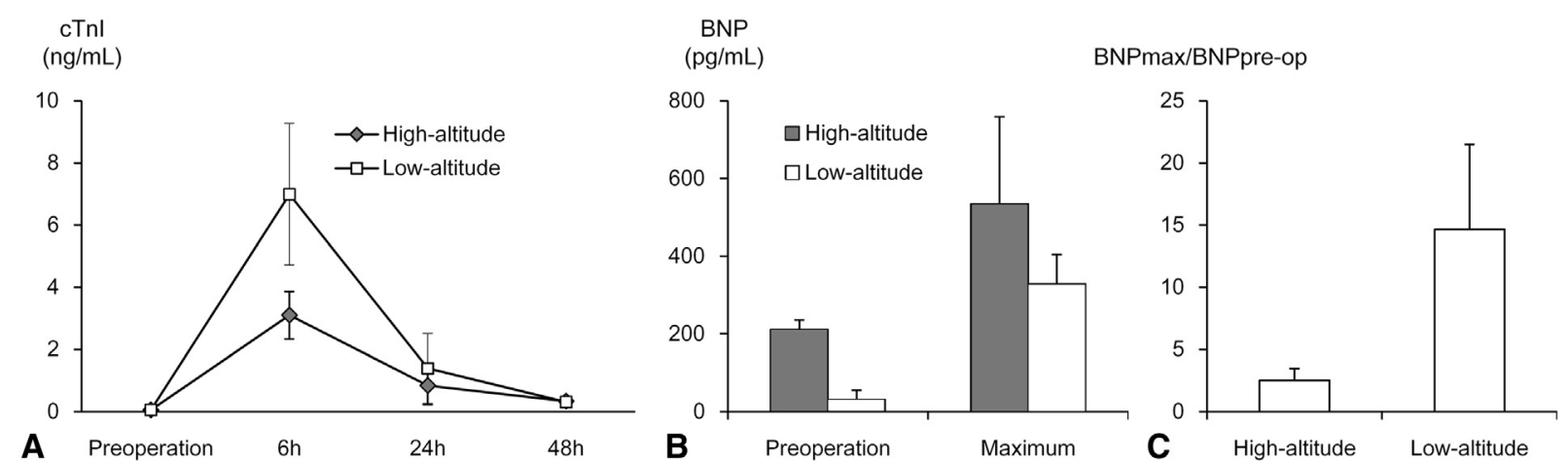

FIGURE 1. Change in molecular marker for myocardial ischemia-reperfusion injury. A, Time course of cardiac troponin I $(c T n I)$ in high- and low-altitude groups. B, Preoperative (pre-op) and maximal brain natriuretic peptide (BNP) concentration in both groups. C, Comparison of increasing $\mathrm{x}$-fold of BNP [(maximum postoperative BNP)/(preoperative BNP)].

Hypoxia affecting the key autophagic mediator, Bnip3. To explore the upstreaming mechanism, the levels of the key autophagic mediator, Bnip3, a hypoxia-inducible Bcl-2 homology 3 domain-containing protein, was evaluated. ${ }^{4}$ The Bnip3 level inclined in both the high- and the low-altitude groups. Its level in the high-altitude group was obviously greater than that in the low-altitude group at both baseline and the ischemia-reperfusion point (Figure 4).

\section{DISCUSSION}

Tibetan patients with CHD could resist ischemiareperfusion injury during cardiac surgery better than those living at sea level, possibly through elevated basal autophagy from chronic hypoxia. To the best of our knowledge, this is the first direct evidence of basal autophagy affecting myocardial ischemia-reperfusion injury in vivo model of humans.

No differences were found in the hematocrit levels between the high- and low-altitude groups, although it was reported that the Tibetan population had lower hemoglobin concentrations than did Han Chinese migrants at high altitude. It seemed counterintuitive to our understanding of the hypoxic induction of red blood cell production. Most existing information on the hematologic characteristics of Tibetans, whether published in the West or in China, was derived from adults. ${ }^{15}$ However, the hemoglobin value of normal Tibetan children was recorded at the lower end of the previously published normal range, no different from that in Han children ${ }^{16}$ and similar to the results from the present study.

Myocardial injury for patients in the low-altitude group was more severe than that in the high-altitude group at the molecular, cellular, and tissue levels. The more impaired left ventricular function in the low-altitude group was hinted at by the more noticeable increasing $\mathrm{x}$-fold of BNP, although no difference was found in the ejection fraction between baseline and the ischemia-reperfusion point or between the 2 groups (data not shown), possibly because of the short ischemia-reperfusion period,
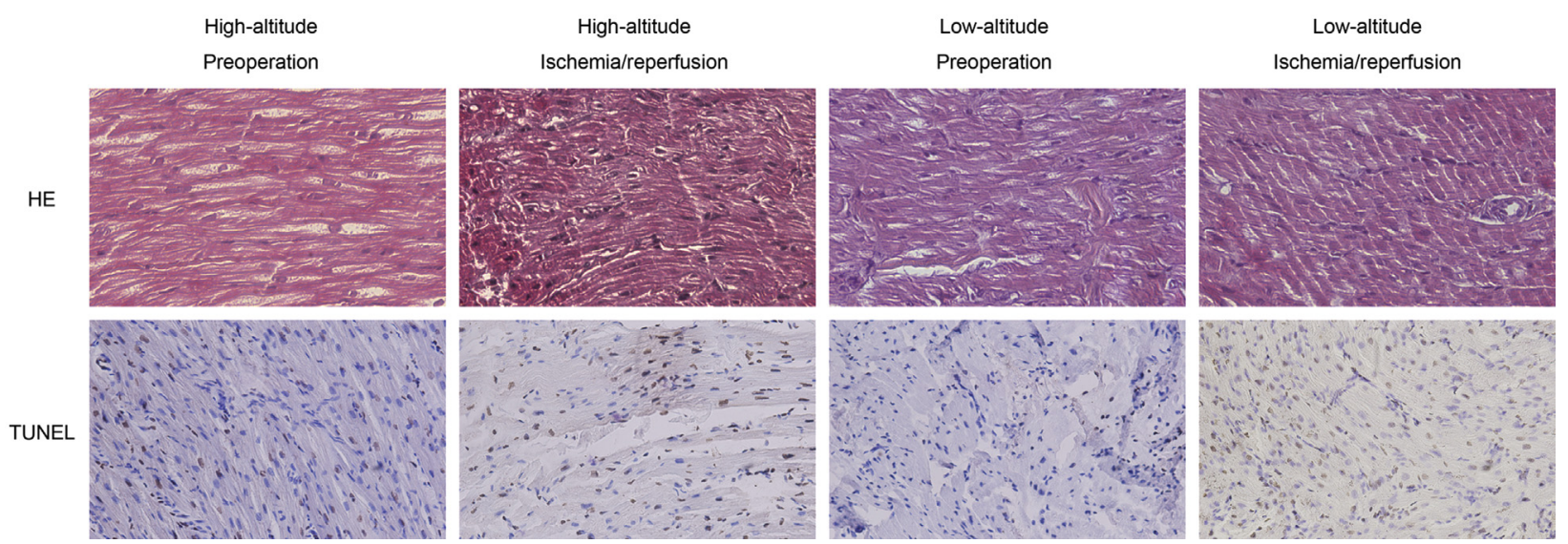

FIGURE 2. Pathologic characteristics of myocardial ischemia-reperfusion. Representative sections from each 1-mm atrial tissue slice were stained with, top row, hematoxylin and eosin $(H E)$ and, bottom row, terminal deoxynucleotidyl transferase-mediated dUTP nick-end labeling assay $(T U N E L)$ (original magnification $\times 20$ ). 


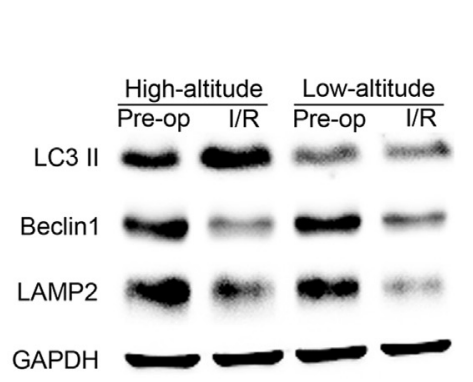

A

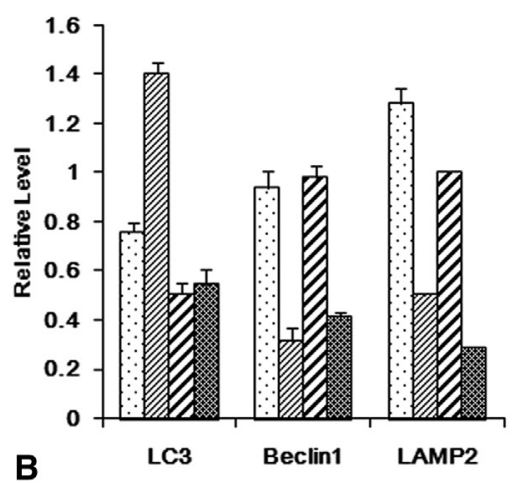

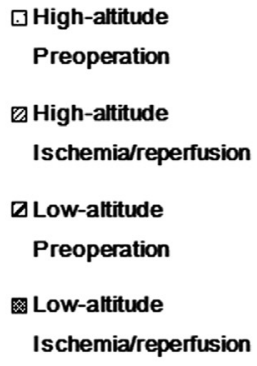

Ischemia/reperfusion

FIGURE 3. Autophagy status before and after ischemia-reperfusion injury. A, Representative immunoblot demonstrating microtubule-associated protein 1 light chain 3 (LC3), beclin1, and lysosomal-associated membrane protein 2 (LAMP2) before ischemia and after ischemia-reperfusion injury. B, Results shown as mean \pm standard error of the mean. Pre-op, Preoperatively; $I / R$, ischemia-reperfusion; GAPDH, glyceraldehyde 3-phosphate dehydrogenase.

cardioprotection measurements, and the minor intracardiac procedure. It was also supported by a greater cTnI level, more severe pathologic changes, and greater apoptosis rate in the low-altitude group than in the high-altitude group.

A large number of recent studies have suggested that autophagy plays a significant and complex role in myocardial ischemia-reperfusion injury. ${ }^{9,10}$ It has been proved by studies of cell and animal models that the autophagy induced through the AMPK/mTOR pathway is beneficial during the ischemic phase. ${ }^{17}$ However, autophagy triggered mainly through the class III PI3K/Beclin1 pathway was detrimental during the reperfusion phase. ${ }^{6}$

In accordance with previous evidence, the total autophagy marked with LC3II increased during ischemiareperfusion in the present study. The Beclin1 levels decreased, in contrast to the findings from animal experiments. ${ }^{6}$ Compared with the long ischemic time (generally $\geq 30$ minutes) in animal experiments, we used only about 20 minutes of aortic clamping time with hyperkalemic cold blood cardioplegia. The existence of a direct correlation between ischemia severity and the extent of autophagy was confirmed during the reperfusion phase. ${ }^{18}$ Hence, activation of autophagy induced through the class III PI3K/Beclin1 pathway during reperfusion might have been modest in our study. Moreover, the basal Beclin1 level in both groups was equal, and the trend during ischemiareperfusion was similar, hinting that beclin1 levels depend on the severity of ischemia but not the presence of chronic hypoxia.

Thus, the autophagy marked with LC3II was derived mainly from the basal status and that induced by ischemia and one of the main possible reasons for the attenuated myocardial ischemia-reperfusion injury. Hypoxia at a high altitude could induce a high expression of Bnip3 by way of the hypoxia-inducible factor 1 binding to the hypoxia-inducible factor 1 response element in the Bnip3 promoter directly, ${ }^{19,20}$ and Bnip3 can directly bind Ras homolog enriched in brain (Rheb), a Ras-related small guanosine triphosphatase, to inhibit the mTOR pathway and induce autophagy. ${ }^{4,21}$ Hence, the high basal status of autophagy in the high-altitude residents probably resulted from the high expression of Bnip3. In addition, autophagy could be induced through the AMPK/mTOR pathway in

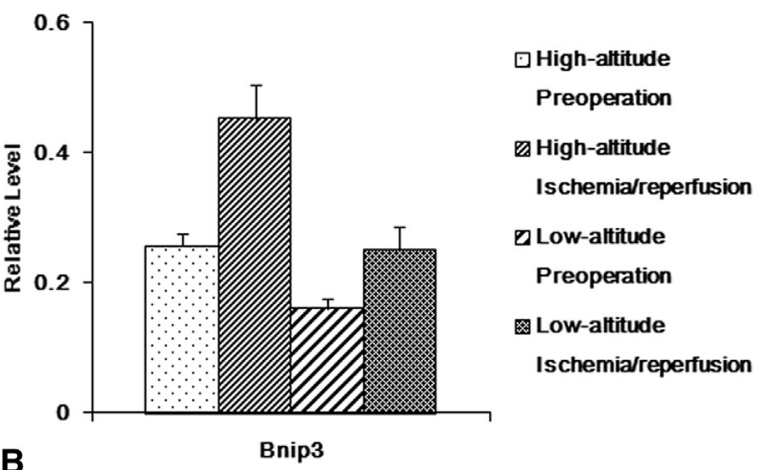

FIGURE 4. Level of BCL2/adenovirus E1B 19-kDa protein-interacting protein 3 (Bnip3) before and after ischemia-reperfusion injury. A, Representative immunoblot of Bnip3 before ischemia and after ischemia-reperfusion injury. B, Results shown as mean \pm standard error of the mean. Pre-op, Preoperatively; $I / R$, ischemia-reperfusion; GAPDH, glyceraldehyde 3-phosphate dehydrogenase. 
both groups. We did not investigate whether chronic hypoxia could make the AMPK/mTOR pathway more sensitive or more efficient during the ischemic phase. Elucidation of the relative contributions of Bnip3 upregulation and the AMPK pathway to autophagy during myocardial ischemia requires additional study.

The autophagosome clearance was also evaluated, because it was thought to be impaired, although cardiomyocyte autophagy was upregulated as a stress response mechanism. Rapid reperfusion-induced decline in LAMP2, a protein critical for autophagosome-lysosome fusion, could impair autophagosome processing, with increased reactive oxygen species generation and mitochondrial permeabilization, thereby provoking cardiomyocyte death. $^{22}$ In the present study, autophagosome clearance in the high-altitude group were still stronger than that in the low-altitude group, just as was the LAMP2 level, although both were impaired, with a declining LAMP2. Less reactive oxygen species production under similar ischemia-reperfusion conditions might result from adaptation to chronic hypoxia in high-altitude residents. $^{23}$

Hence, the attenuated myocardial ischemia-reperfusion injury observed in the high-altitude residents possibly resulted from the high base level of autophagy induced by chronic hypoxia. Improving the basal autophagy status might thus be a good approach to decreasing ischemiareperfusion injury.

The present study had several limitations that should be considered. First, it was limited by the small sample size because of the difficulty of recruiting patients with $\mathrm{CHD}$ living at a high altitude. However, that was resolved to some extent by the adoption of criteria controlling for other factors affecting autophagy and direct myocardial injury. Second, some genetic differences might have been present between the 2 ethnic groups. ${ }^{24}$ Third, tissue samples were taken from the atrial incision instead of ventricular tissue, in accordance with ethical requirements. This could have resulted in additional myocardial injury from intraoperative retraction. For the same reasons, taking more tissue specimens was not done at the moment of aortic opening. In addition, we did not test whether these benefits could extend to other groups with chronic hypoxia, such as patients with chronic obstructive pulmonary disease, allowing them to undergo valve surgery or coronary artery bypass grafting, and patients with cyanotic heart disease. It would be difficult to evaluate the cardioprotection induced by chronic hypoxia in these groups with age-related autophagy dysfunction or directly impaired myocardium. These limitations could be resolved in animal models with autophagy intervention in the future.

We wish to thank Yao Zhang, Associated Professor, Department of Epidemiology, Clinic Epidemiology Center,
Third Military Medical University, for the statistical analysis, and the members of the clinical research assistant team (Caijing Peng, Mingxiang Chen, Jianying Deng, Ye Yuan, Xiangli Liao, Xiaoying Zhao, Wenshuai Tang, Jun Lei, Jing Chen, and Xue Bai).

\section{References}

1. Miao CY, Zuberbuhler JS, Zuberbuhler JR. Prevalence of congenital cardiac anomalies at high altitude. J Am Coll Cardiol. 1988;12:224-8.

2. Weitz CA, Garruto RM. A comparative analysis of arterial oxygen saturation among Tibetans and Han born and raised at high altitude. High Altitude Med Biol. 2007;8:13-26.

3. Zhou ZN, Zhuang JG, Wu XF, Zhang Y, Cherdrungsi P. Tibetans retained innate ability resistance to acute hypoxia after long period of residing at sea level J Physiologic Sci. 2008;58:167-72.

4. Li Y, Wang Y, Kim E, Beemiller P, Wang CY, Swanson J, et al. Bnip3 mediates the hypoxia-induced inhibition on mammalian target of rapamycin by interacting with Rheb. J Biol Chem. 2007;282:35803-13.

5. Dong Y, Undyala VV, Gottlieb RA, Mentzer RM Jr, Przyklenk K. Autophagy: definition, molecular machinery, and potential role in myocardial ischemia-reperfusion injury. J Cardiovasc Pharmacol Ther. 2010; 15:220-30.

6. Matsui Y, Takagi H, Qu X, Abdellatif M, Sakoda H, Asano T, et al. Distinct roles of autophagy in the heart during ischemia and reperfusion: roles of AMP-activated protein kinase and Beclin 1 in mediating autophagy. Circ Res. 2007; 100:914-22.

7. Takagi H, Matsui Y, Sadoshima J. The role of autophagy in mediating cell survival and death during ischemia and reperfusion in the heart. Antioxid Redox Signal. 2007;9:1373-81.

8. Gustafsson AB, Gottlieb RA. Eat your heart out: role of autophagy in myocardial ischemia/reperfusion. Autophagy. 2008;4:416-21.

9. Matsui Y, Kyoi S, Takagi H, Hsu CP, Hariharan N, Ago T, et al. Molecular mechanisms and physiological significance of autophagy during myocardial ischemia and reperfusion. Autophagy. 2008;4:409-15.

10. Huang C, Yitzhaki S, Perry CN, Liu W, Giricz Z, Mentzer RM Jr, et al Autophagy induced by ischemic preconditioning is essential for cardioprotection. J Cardiovasc Transl Res. 2010;3:365-73.

11. Hamacher-Brady A, Brady NR, Gottlieb RA. Enhancing macroautophagy protects against ischemia/reperfusion injury in cardiac myocytes. $J$ Biol Chem 2006;281:29776-87.

12. Zingarelli B, Salzman AL, Szabo C. Genetic disruption of poly (ADP-ribose) synthetase inhibits the expression of P-selectin and intercellular adhesion molecule-1 in myocardial ischemia/reperfusion injury. Circ Res. 1998;83: 85-94.

13. Sinha AM, Breithardt OA, Schmid M, Stellbrink C. Brain natriuretic peptide release in cardiac surgery patients. Thorac Cardiovasc Surg. 2005;53: 138-43.

14. Mitchell J, Webb ST. Is brain natriuretic peptide a marker for adverse postoperative outcomes in patients undergoing cardiac surgery? Interact Cardiovasc Thorac Surg. 2011;12:467-72.

15. Garruto RM, Chin CT, Weitz CA, Liu JC, Liu RL, He X. Hematological differences during growth among Tibetans and Han Chinese born and raised at high altitude in Qinghai, China. Am J Phys Anthropol. 2003; 122:171-83

16. Wu T, Wang X, Wei C, Cheng H, Li Y, Ge D, et al. Hemoglobin levels in Qinghai-Tibet: different effects of gender for Tibetans vs. Han. J Appl Physiol (1985). 2005;98:598-604.

17. Takagi H, Matsui Y, Hirotani S, Sakoda H, Asano T, Sadoshima J. AMPK mediates autophagy during myocardial ischemia in vivo. Autophagy. 2007;3: 405-7.

18. Sciarretta S, Hariharan N, Monden Y, Zablocki D, Sadoshima J. Is autophagy in response to ischemia and reperfusion protective or detrimental for the heart? Pediatr Cardiol. 2011;32:275-81.

19. Hamacher-Brady A, Brady NR, Logue SE, Sayen MR, Jinno M, Kirshenbaum LA, et al. Response to myocardial ischemia/reperfusion injury involves Bnip3 and autophagy. Cell Death Differ. 2007;14:146-57.

20. Ma X, Godar RJ, Liu H, Diwan A. Enhancing lysosome biogenesis attenuates BNIP3-induced cardiomyocyte death. Autophagy. 2012;8: 297-309. 
21. Sciarretta S, Zhai P, Shao D, Maejima Y, Robbins J, Volpe M, et al. Rheb is a critical regulator of autophagy during myocardial ischemia: pathophysiological implications in obesity and metabolic syndrome. Circulation. 2012;125:1134-46.

22. Ma X, Liu H, Foyil SR, Godar RJ, Weinheimer CJ, Hill JA, et al Impaired autophagosome clearance contributes to cardiomyocyte death in ischemia/reperfusion injury. Circulation. 2012;125:3170-81.
23. Ali SS, Hsiao M, Zhao HW, Dugan LL, Haddad GG, Zhou D. Hypoxia-adaptation involves mitochondrial metabolic depression and decreased ROS leakage. PLoS One. 2012;7:e36801.

24. Peng Y, Yang Z, Zhang H, Cui C, Qi X, Luo X, et al. Genetic variations in Tibetan populations and high-altitude adaptation at the Himalayas. Mol Biol Evol. 2011;28:1075-81. 